\title{
Geochemical characteristics and reservoir forming mechanism of large gas field with high water cut of the Upper Triassic Xujiahe Formation in Sichuan Basin,China
}

\author{
XIE ZENGYE ${ }^{1,2}$, YANG CHUNLONG ${ }^{1,2}$, LI JiAN ${ }^{1,2}$, JiN HUI \\ ${ }^{1,2}$, ZHANG Lu $^{1,2}$, GuO JiANYING ${ }^{1,2}$, HaO CuiGuO ${ }^{1,2}$ \\ ${ }^{1}$ Research Institute of Petroleum Exploration \& Development, \\ Langfang, 065007, China \\ ${ }^{2}$ Laboratory of Gas Reservoir Formation \& Development, \\ CNPC, Langfang, 065007, China
}

A series of large tight sandstone gas fields such as Anyue, Guang 'an, Hechuan and Penglai have been found in the Upper Triassic Xujiahe Formation in central Sichuan Basin of China, where the gas generation intensity is less than $20 \times 10^{8} \mathrm{~m}^{3} / \mathrm{km}^{2}$, forming an area of nearly one trillion cubic meters of natural gas reserves. However, the gas reservoir is generally water-bearing, and the gas saturation is mainly $50 \%$ $\sim 65 \%$. Multiple geochemical evidences indicate that tight sandstone gas is mainly concentrated by near source rock. The on-line dynamic simulation of gas-drive water process of Xujiahe Formation tight sandstone under different pressures is carried out using the simulation experimental equipment based on the combination of low-field NMR and highpressure displacement equipment. The results show that: (1) the main reservoir that determines the gas saturation of Xujiahe Formation is the reservoir space with a pore radius of $0.1 \sim 10 \mu \mathrm{m}$. (2) The gas saturation is mainly controlled by the pore radius that larger than $1 \mu \mathrm{m}$ when the porosity and permeability are similar, and the larger the proportion of large pore, the higher the gas saturation. (3)The tight sandstone of Xujiahe Formation reaches $70 \%$ of the total gas saturation at $3 \sim 5.5 \mathrm{MPa}$ filling pressure, and then the gas saturation increases slowly and the total amount is small with the increase of filling pressure. (4)"Coupling of small pressure difference driven $(3 \sim 5.5 \mathrm{MPa})$ and relatively large pore $(>0.1 \mu \mathrm{m})$ space reservoir" is an important factor for the formation of large and medium sized gas fields with high water saturation in tight sandstone of low gas generation intensity region. The research results will provide theoretical and technical support for the exploration of tight sandstone gas in Xujiahe Formation and other areas.

This study is Supported by National Science \& Technology Major Project (2016ZX05007-003) ; China Petroleum Science and Technology Project (2019B-0605). 